\title{
Energy estimates for Galerkin semidiscretizations of time domain boundary integral equations
}

\author{
Francisco--Javier Sayas*
}

January 19, 2018

\begin{abstract}
In this paper we present a battery of results related to how Galerkin semidiscretization in space affects some formulations of wave scattering and propagation problems when retarded boundary integral equations are used.
\end{abstract}

\section{Introduction}

In this paper we present several results concerning the effect of Galerkin semidiscretization in space when applied to formulations where time domain boundary integral equations are used. The focus will be set on energy conservation properties (or lack thereof) and the model equation will be the acoustic wave equation in two and three dimensions.

While frequency domain boundary integral equations are very often used for scattering problems and for construction of absorbing boundary conditions, their time domain counterparts have enjoyed a more limited success. The work of Alain Bamberger and Toung Ha-Duong [3]- 4] in the mid-80s sparked theoretical and practical activity concerning time domain integral equations for acoustics, elastodynamics and electromagnetism. A detailed account of this early work can be found in the review articles [14] and [11]. It is nowadays well understood that some of the apparently unsurmountable difficulties in the development stable discretizations of the time domain integral equations are related to the correct approximation of integrals and that these equations can be advantageously used in situations where frequency domain formulations are not competitive (scattering of frequency rich noise) or applicable (coupling with nonlinear problems). In this paper we will study semidiscretization in space with general Galerkin schemes, which can be complemented either with Galerkin in time discretization (following the philosophy of [3] and its consequences) or with Convolution Quadrature in time (following the seminal work of Christian Lubich [21] and its development in [5], 8], [24], [7]).

From the point of view of the type of problems we are going to study, we can classify them in two groups:

${ }^{*}$ Department of Mathematical Sciences, University of Delaware, Newark DE 19716, USA - e-mail: fjsayas@math.udel.edu 
(a) Scattering of acoustic waves by impenetrable obstacles. This leads to an exterior wave equation with Dirichlet or Neumann boundary conditions on the surface of the obstacles. Instead of dealing with the traditional (and more practical) problem of an incident plane or spherical wave (that has no finite energy), we will study the effect of the scattering of a free wave that propagates from some compactly supported initial conditions.

(b) Propagation of waves originated in a locally non-homogeneous medium surrounded by a homogeneous medium. In this case, we will also follow the propagation of compactly supported initial data when we surround the computational domain by an artificial boundary where we impose non-local boundary conditions using time domain integral equations that give an exact expression of the corresponding SteklovPoincaré operators.

In order to present the techniques and results in a clear way we will first focus on the problem of scattering of waves by a sound-soft obstacle, formulated using a single-layer potential representation (i.e., with an indirect boundary integral method). We will show that any Galerkin semidiscretization in space of the corresponding retarded integral equation is stable from the point of view that a certain energy functional is preserved over time. In fact, we will show that the total energy that is conserved has to be computed counting potential and kinetic energy in the exterior and interior of the obstacle, thus giving theoretical evidence of the fact that energy is leaked to the interior of the scatterer, but the total balance of energy is still correct. In a second step, we introduce the second class of problems where a locally non-homogeneous medium is surrounded with an artificial boundary (that also surrounds the support of the initial data) and we follow the propagation of these initial data when we discretize in space with a Galerkin scheme both for the variational formulation of the wave equation in the computational domain and for a system of two retarded boundary integral equations that are coupled with the interior wave equation to construct an absorbing boundary condition on the artificial interface. At the discrete level, this can be understood as a time domain coupling of Boundary and Finite Elements (BEM-FEM). The result that we show is very similar to the one for the scattering problem: energy is conserved as a function of time (independently of Galerkin discretization) as long as we count the energy of a ghost wave in the computational domain -the energy of this interior wave has to be computed using the material properties of the surrounding medium and is added to the natural energy of the total wave in the interior and exterior domains.

Once these two model situations have been analyzed, we present two very simple extensions where the same techniques apply to establish similar results. The first extension deals with the scattering of a sound-hard obstacle formulated with a double-layer acoustic potential. The second one is the construction of a tighter integral absorbing boundary condition using two integral equations but only one boundary unknown. We next show that there are formulations where energy is not conserved after space discretization. Two of them are explored: the use of a direct integral formulation (based on Kirchhoff's formula) for the sound-soft scattering problem and the construction of absorbing boundary conditions with only one integral equation. While the negative results do not imply that these discretizations are unstable, they might indicate some undesirable effects. Actually, 
the direct integral formulation for the sound-soft scattering problem, fully discretized with a Galerkin method in space and Convolution Quadrature (CQ) in time, can be analyzed [6] (See below for more on this.) As for the one-equation absorbing boundary condition, at present time there seems to be insufficient theoretical and practical evidence to suggest convergence or lack thereof. The extension of all the results in this paper to linear elastic waves is straightforward. Extension to other types of elastic waves (viscoelastic or poroelastic, for instance) and to electromagnetic waves will possibly require more effort.

Apart from the inherent interest of having theoretical evidence of energy preservation after space discretization of some integral and integro-differential model problems for which this energy conservation property does not seem to be evident, the type of results that we prove in this paper has some other applications. On the one hand, the transformation of the semidiscrete equations to a transmission problem is allowing us to provide an analysis of the CQ-BEM for some scattering problems [6]. Up to the present time, analysis of discrete methods for retarded integral equations was based on estimates in the Laplace domain. The dynamical system approach that we develop in this paper can be used to obtain better estimates of the effect of space discretization and approximation of data in some retarded integral equations. It is also our belief that these techniques will be advantageous for some kind of analysis of fully discrete BEM-FEM schemes using CQ. In this sense, this paper can be taken as the time domain counterpart of what was done in [20] in the Laplace domain and we hope to be able to build on these two articles for the complete analysis of the fully discrete method.

This paper offers a novel approach to the analysis of time-domain integral equations and their semidiscretization in space. Theoretical study of retarded integral equations is almost exclusively carried out in the Laplace domain. Invertibility of the corresponding boundary integral equations is established in the resolvent set of the Laplacian and bounds depending on the Laplace transformed parameter are used to move back to the time domain. While this proved to be an extremely powerful approach, leading to solid estimates and useful in the analysis of CQ-type time discretization, it is also clear that some properties are lost in the process of transforming back and forth to the Laplace domain and the some of the functional spaces that this kind of analysis impose might not be optimal. This paper offers a complementary point of view using elementary techniques from the theory of evolution equations based on strongly continuous semigroups (cf. [12], [18]). The main idea is recasting the semidiscrete problem as a Cauchy problem for an equation of the second order associated to an unbounded operator. Once the problem is reexpressed as a second order equation in time, we use basic estimates in the simplest possible setting (Hilbert space theory and a coincidence of two of the four possible spaces that appear in the abstract formulation) in order to simply derive the energy conservation property. The kind of unbounded operator that will appear in this formulation is very closely related to the exotic transmission problems that Antonio Laliena and the author of this paper devised for the analysis of CQ schemes applied to non-trivial acoustic scattering problems in [20]. The surprisingly simple (and quite effective) idea consists of dealing with the different aspects of Galerkin discretization (the fact that the unknown is sought in a discrete space and the fact that the corresponding equation is tested with the same space) as non-standard transmission conditions.

The paper is organized as follows. In Section 2 we present the indirect indirect formula- 
tion of the sound-soft scattering problem and its Galerkin semidiscretization, and we state the energy conservation result for this problem. The proof of this result is carried out in Section 3 by using the techniques that have been described before, and taking advantage of well known results for the single layer potential associated to the Yukawa (reactiondifussion) equation. In Section 4 we present a boundary integral absorbing boundary condition for a wave propagation problem in free space and its Galerkin (BEM-FEM) discretization, and we state the corresponding energy estimate. The proof of this result is the content of Section 5. Section 6 presents two easy generalizations: indirect formulation for sound-hard scattering and a different absorbing boundary condition. In Section 7 we give two partially negative results, showing that space discretization does not lead to energy conservation either in a direct formulation of a scattering problem or a one-equation absorbing boundary condition. Finally, Section 8 includes the abstract frame of Cauchy problems for equations of the second order associated to a class of unbounded operators. These results are easy modifications of theorems that are well known in the literature of $C_{0}$-semigroups and are presented here in order to have an easy reference in the same language that we are using in the paper.

Notational foreword. Basic elementary results on Sobolev spaces will be assumed throughout without specific reference. All of them can be found in any text on the subject ([1] for instance). Given an open set $U$ with Lipschitz boundary, we will consider the spaces $L^{2}(U)$ and $H^{m}(U)$ for $m \geq 1$. The $L^{2}(U)$-norm will be denoted $\|\cdot\|_{U}$ and the $H^{1}(U)$-norm $\|\cdot\|_{1, U}$. Two fractional Sobolev spaces will be used on the boundary $H^{ \pm 1 / 2}(\partial U)$. The space $H_{0}^{1}(U)$ is the kernel of the trace operator $H^{1}(U) \rightarrow H^{1 / 2}(\partial U)$. The characteristic function of $U$ will be denoted $\chi_{U}$.

Although the geometric layout of the different parts of this article will vary, in all cases, there will be a bounded open set $\Omega$, with exterior $\Omega^{+}:=\mathbb{R}^{d} \backslash \bar{\Omega}$ and common Lipschitz boundary $\Gamma:=\partial \Omega=\partial \Omega^{+}$. There are two possible traces on $\Gamma$, which will be respectively denoted $\gamma^{+}: H^{1}\left(\Omega^{+}\right) \rightarrow H^{1 / 2}(\Gamma)$ and $\gamma^{-}: H^{1}(\Omega) \rightarrow H^{1 / 2}(\Gamma)$. Whenever there is a single trace or functions are exclusively defined in the interior domain, the superscript will be dropped.

For functions of space and time variables $u(\mathbf{x}, t)$, we will often employ the notation of theory of evolution equations, where only the time variable is displayed. This amounts to considering functions $u:[0, T] \rightarrow X$, where $X$ is a space of functions of the $\mathbf{x}$ variable. With this notation, $\dot{u}$ and $\ddot{u}$ denote the first and second $t$-derivatives of $u(t)$.

\section{Single layer potentials for sound-soft scattering}

Consider a bounded open set $\Omega \subset \mathbb{R}^{d}$, with Lipschitz boundary $\Gamma:=\partial \Omega$ such that $\Omega^{+}:=\mathbb{R}^{d} \backslash \bar{\Omega}$ is connected $\left(\Omega\right.$ does not need to be connected though). Let $u_{0}$ and $v_{0}$ be given functions such that $\mathcal{O}:=\operatorname{supp} u_{0} \cup \operatorname{supp} v_{0}$ is compact and $\mathcal{O} \cap \bar{\Omega}=\emptyset$. We consider the following problem of scattering of acoustic waves by a sound-soft obstacle: we look 
for $u:[0, \infty) \rightarrow H^{1}\left(\Omega^{+}\right)$such that for all $t>0$

$$
\begin{aligned}
\ddot{u}=\Delta u & \text { in } \Omega^{+}, \\
u=0 & \text { on } \Gamma,
\end{aligned}
$$

and the initial conditions $\left(\right.$ on $\left.\Omega^{+}\right)$

$$
u(0)=u_{0}, \quad \dot{u}(0)=v_{0}
$$

are satisfied. We will additionally assume that $u_{0} \in H_{0}^{2}\left(\Omega^{+}\right)$and $v_{0} \in H_{0}^{1}\left(\Omega^{+}\right)$. Let $R>0$ be such that

$$
\bar{\Omega} \cup \mathcal{O} \subset B(0 ; R):=\left\{\mathbf{x} \in \mathbb{R}^{d}:|\mathbf{x}|<R\right\} .
$$

Because of the finite speed of propagation of waves, solutions of problem (11)-(2) are supported in $B(0 ; R+T)$ for all $t \in[0, T]$.

The solution of (11)-(2) will be decomposed as the sum of a free (or incident) wave and a scattered wave. To define the free wave we need to extend the initial data by zero to the interior of the scatterer:

$$
\mathrm{E} v:= \begin{cases}v & \text { in } \Omega^{+} \\ 0 & \text { in } \Omega .\end{cases}
$$

The free wave is the solution of the wave equation in $\mathbb{R}^{d}$ for $t>0$

$$
\ddot{u}^{\text {free }}=\Delta u^{\text {free }}
$$

satisfying initial conditions

$$
u^{\text {free }}(0)=\mathrm{E} u_{0}, \quad \dot{u}^{\text {free }}(0)=\mathrm{E} v_{0} .
$$

The solution to this problem satisfies

$$
u^{\text {free }} \in \mathcal{C}^{2}\left([0, \infty) ; L^{2}\left(\mathbb{R}^{d}\right)\right) \cap \mathcal{C}^{1}\left([0, \infty) ; H^{1}\left(\mathbb{R}^{d}\right)\right) \cap \mathcal{C}\left([0, \infty), H^{2}\left(\mathbb{R}^{d}\right)\right)
$$

and $\operatorname{supp} u^{\text {free }}(t) \subset B(0 ; R+t)$ for all $t$. The scattered wave is the difference $u-u^{\text {free }}$.

Remark 2.1. For the kind of arguments that we are going to develop, we will assume that the free wave is known. Section 4 deals with a semidiscrete version of a problem that generalizes (4)-(5), leading to algorithms to compute the propagation of compactly supported initial data in free space. In applications, the free or incident wave is actually known. Typically the free wave is the solution of a non-homogeneous wave equation and has singularities at some points away from the scatterer. Other practical incident waves include plane waves that are not compactly supported at any given time and have infinite energy. Because our interest lies in how discretization affects the integral model used to represent $u-u^{\text {free }}$, the use of free waves as those of (4)-(5) fulfills our needs.

The scattered wave $u-u^{\text {free }}$ can be represented with a single layer retarded potential 3]. For $(\mathbf{x}, t) \in \Omega^{+} \times[0, \infty)$ and a given density $\psi:[0, \infty) \rightarrow H^{-1 / 2}(\Gamma)$ the single layer potential is defined as

$$
(\mathcal{S} * \psi)(\mathbf{x}, t)= \begin{cases}\int_{\Gamma} \frac{\psi(\mathbf{y}, t-|\mathbf{x}-\mathbf{y}|)}{4 \pi|\mathbf{x}-\mathbf{y}|} \mathrm{d} \Gamma(\mathbf{y}) & (\text { when } d=3), \\ \int_{\Gamma} \int_{0}^{t-|\mathbf{x}-\mathbf{y}|} \frac{\psi(\mathbf{y}, \tau)}{\sqrt{(t-\tau)^{2}-|\mathbf{x}-\mathbf{y}|^{2}}} \mathrm{~d} \Gamma(\mathbf{y}) \mathrm{d} \tau & (\text { when } d=2) .\end{cases}
$$


The integral forms in (17) are only valid for smooth densities. Weak forms of the potentials have to be used in general (see [19] for full justification in the three dimensional case). We will keep the convolutional notation for the layer potential $\mathcal{S} * \psi$ in order to distinguish time domain potentials and operators from similar entities for steady state problems. Let

$$
\mathcal{V} * \psi:=\gamma(\mathcal{S} * \psi)
$$

be the corresponding single layer integral operator (see [3] and [14]). The indirect representation of the scattered field looks for a density $\psi: \mathbb{R} \rightarrow H^{-1 / 2}(\Gamma)$ such that

$$
\psi \equiv 0 \text { in }(-\infty, 0) \quad \mathcal{V} * \psi+\gamma u^{\text {free }}=0
$$

(this is an equation on $\Gamma \times[0, \infty)$ ) and then represents the total wave by

$$
u=\mathcal{S} * \psi+u^{\text {free }} .
$$

Equation (8) is now approximated with a Galerkin method only in the space variable. To do that, we choose a sequence of finite dimensional spaces

$$
X_{h} \subset H^{-1 / 2}(\Gamma),
$$

approximate (88) by the problem

$$
\left[\begin{array}{l}
\psi_{h}: \mathbb{R} \rightarrow X_{h}, \quad \psi_{h} \equiv 0 \text { in }(-\infty, 0) \\
\left\langle\mu_{h}, \mathcal{V} * \psi_{h}+\gamma u^{\text {free }}\right\rangle_{\Gamma}=0 \quad \forall \mu_{h} \in X_{h} \quad \forall t
\end{array}\right.
$$

and write an approximation of the total wave as

$$
u_{h}:=\mathcal{S} * \psi_{h}+u^{\text {free }}
$$

The angled bracket in (10) denotes the $H^{-1 / 2}(\Gamma) \times H^{1 / 2}(\Gamma)$ duality product. Note that $\operatorname{supp} u_{h}(t) \subset B(\mathbf{0} ; R+t)$ for all $t$, because the layer potential $\mathcal{S} * \psi$ propagates a wave from $\Gamma$ at unit velocity starting at time $t=0$.

Remark 2.2. Problem (10) is a system of functional equations. Let us assume that $X_{h} \subset L^{\infty}(\Gamma)$ and that $\left\{N_{j}: j=1, \ldots m\right\}$ is a basis of $X_{h}$. We can then write the unknown density as

$$
\psi_{h}(\mathbf{y}, t)=\sum_{j=1}^{M} \psi_{j}(t) N_{j}(\mathbf{x}) \quad \psi_{j}: \mathbb{R} \rightarrow \mathbb{R}, \quad \operatorname{supp} \psi_{j} \subset(0, \infty) .
$$

In the three dimensional case, (10) is then equivalent to

$$
\begin{array}{r}
\sum_{j=1}^{M} \int_{\Gamma} \int_{\Gamma} \frac{N_{j}(\mathbf{y}) N_{i}(\mathbf{x})}{4 \pi|\mathbf{x}-\mathbf{y}|} \psi_{j}(t-|\mathbf{x}-\mathbf{y}|) \mathrm{d} \Gamma(\mathbf{x}) \mathrm{d} \Gamma(\mathbf{y})=-\int_{\Gamma} N_{i}(\mathbf{x}) u^{\text {free }}(\mathbf{x}, t) \mathrm{d} \Gamma(\mathbf{x}) \\
i=1, \ldots, M .
\end{array}
$$

This system of functional equations include integrated delays of all the unknowns. 
We are now in conditions to state the main theorem of this part of the article, showing energy conservation and regularity in time for the semidiscrete total wave field. The proof of this theorem will be given in Section 3 .

Theorem 2.1. The semidiscrete total field $u_{h}$ and the associated density $\psi_{h}$, given by (10) -(11), satisfy

$$
\begin{aligned}
& u_{h} \in \mathcal{C}^{2}\left([0, \infty) ; L^{2}\left(\mathbb{R}^{d}\right)\right) \cap \mathcal{C}^{1}\left([0, \infty) ; H^{1}\left(\mathbb{R}^{d}\right)\right) \\
& \psi_{h} \in \mathcal{C}\left([0, \infty) ; H^{-1 / 2}(\Gamma)\right) .
\end{aligned}
$$

Moreover, the energy

$$
\frac{1}{2}\left\|\nabla u_{h}(t)\right\|_{\mathbb{R}^{d}}^{2}+\frac{1}{2}\left\|\dot{u}_{h}(t)\right\|_{\mathbb{R}^{d}}^{2}
$$

is constant over time.

Let us emphasize that the exact total wave field $u$ is only defined in $\Omega^{+}$and that it can be extended by zero to the interior of the obstacle. The semidiscrete total wave field $u_{h}$ is however defined in $\mathbb{R}^{d}$ and the property of energy conservation is proved with integration over $\mathbb{R}^{d}$ and not only over $\Omega^{+}$. This shows that Galerkin discretization leaks part of the energy to the interior of the obstacle, although the total energy is still constant.

\section{Proof of Theorem 2.1}

Notation. The jump of the trace across $\Gamma$ is denoted

$$
\llbracket \gamma u \rrbracket:=\gamma^{-} u-\gamma^{+} u
$$

Weak normal derivatives on $\Gamma$ can be defined using Green's formula. Given an open bounded set $\mathbb{B}$ that contains $\bar{\Omega}$, we can define $\partial_{\nu}^{ \pm} u$ for any $u \in H^{1}(\mathbb{B} \backslash \Gamma)$ such that $\Delta u \in L^{2}(\mathbb{B} \backslash \Gamma)$ with the formulas:

$$
\begin{aligned}
& \left\langle\partial_{\nu}^{-} u, \gamma^{-} v\right\rangle_{\Gamma}=(\nabla u, \nabla v)_{\Omega^{-}}+(\Delta u, v)_{\Omega^{-}} \quad \forall v \in H^{1}\left(\Omega^{-}\right), \\
& \left\langle\partial_{\nu}^{+} u, \gamma^{+} v\right\rangle_{\Gamma}=-(\nabla u, \nabla v)_{\mathbb{B} \cap \Omega^{+}}-(\Delta u, v)_{\mathbb{B} \cap \Omega^{+}} \quad \forall v \in H_{\partial \mathbb{B}}^{1}\left(\mathbb{B} \cap \Omega^{+}\right),
\end{aligned}
$$

where

$$
H_{\partial \mathbb{B}}^{1}\left(\mathbb{B} \cap \Omega^{+}\right)=\left\{v \in H^{1}\left(\mathbb{B} \cap \Omega^{+}\right): \gamma_{\partial \mathbb{B}} v=0\right\}
$$

and $\gamma_{\partial \mathbb{B}}$ is the trace operator associated to the boundary of $\mathbb{B}$. It is well known that the definition of the exterior normal derivative is independent of the set $\mathbb{B}$. The jump of the normal derivative $\llbracket \partial_{\nu} u \rrbracket:=\partial_{\nu}^{-} u-\partial_{\nu}^{+} u$ is defined for the same class of functions.

The single layer Yukawa potential. Consider the fundamental solution of the Yukawa operator $u \mapsto-\Delta u+u$ :

$$
E(\mathbf{x}, \mathbf{y}):= \begin{cases}\frac{e^{-|\mathbf{x}-\mathbf{y}|}}{4 \pi|\mathbf{x}-\mathbf{y}|} & (\text { when } d=3) \\ \frac{1}{2 \pi} K_{0}(|\mathbf{x}-\mathbf{y}|) & (\text { when } d=2),\end{cases}
$$


where $K_{0}$ is the modified Bessel function of the second kind and order zero. On the surface/curve $\Xi:=\partial \mathbb{B} \cup \Gamma$, we can define the single layer potential

$$
\mathrm{S} \lambda:=\int_{\Xi} E(\cdot, \mathbf{y}) \lambda(\mathbf{y}) \mathrm{d} \Xi(\mathbf{y})
$$

Using a weak definition of this potential (see [10] or the very general theory developed in $[22])$, we can prove that $\mathrm{S}: H^{-1 / 2}(\Xi) \rightarrow H^{1}\left(\mathbb{R}^{d}\right)$ is bounded, that

$$
\mathrm{V}:=\gamma_{\Xi} \mathrm{S}: H^{-1 / 2}(\Xi) \rightarrow H^{1 / 2}(\Xi)
$$

is bounded and coercive

$$
\langle\lambda, \mathrm{V} \lambda\rangle_{\Xi} \geq C\|\mu\|_{-1 / 2, \Xi}^{2} \quad \forall \lambda \in H^{-1 / 2}(\Xi),
$$

and that $\Delta(\mathrm{S} \lambda)=\mathrm{S} \lambda$ in $\mathbb{R}^{d} \backslash \Xi$ for all $\lambda$. Also, if we write $\lambda=\left(\lambda_{\Gamma}, \lambda_{\partial}\right) \in H^{-1 / 2}(\Xi) \cong$ $H^{-1 / 2}(\Gamma) \times H^{-1 / 2}(\partial \mathbb{B})$, then $\llbracket \partial_{\nu}(\mathrm{S} \lambda) \rrbracket=\lambda_{\Gamma}$ (the jump is defined across $\left.\Gamma\right)$.

A transmission problem. The single layer retarded potential satisfies the following properties [3]

$$
\llbracket \gamma(\mathcal{S} * \psi) \rrbracket=0 \quad \llbracket \partial_{\nu}(\mathcal{S} * \psi) \rrbracket=\psi \quad(\mathcal{S} * \psi)(0)=0 \quad \frac{d}{d t}(\mathcal{S} * \psi)(0)=0 .
$$

For given initial data with compact support we can choose $R>0$ so that (3) is satisfied. Since the speed of propagation of $u^{\text {free }}$ and $\mathcal{S} * \psi_{h}$ is the same,

$$
\operatorname{supp} u_{h}(t) \subset \mathbb{B}:=B(\mathbf{0} ; R+T) \quad \forall t \in[0, T] .
$$

Noticing that

$$
\llbracket \gamma u_{h} \rrbracket=\llbracket \gamma\left(\mathcal{S} * \psi_{h}\right) \rrbracket+\llbracket \gamma u^{\text {free }} \rrbracket=0,
$$

it follows that, restricted to the time interval $[0, T]$, the function defined by (10)-(11) can be understood as $u_{h}:[0, T] \rightarrow H^{1}(\mathbb{B} \backslash \Gamma)$ that solves the wave propagation problem:

$$
\begin{array}{r}
\ddot{u}_{h}=\Delta_{ \pm} u_{h}, \\
\llbracket \gamma u_{h} \rrbracket=0, \\
\gamma u_{h} \in X_{h}^{\circ}, \\
\llbracket \partial_{\nu} u_{h} \rrbracket \in X_{h}, \\
\gamma_{\partial \mathbb{B}} u_{h}=0,
\end{array}
$$

with initial conditions

$$
u_{h}(0)=\mathrm{E} u_{0} \quad \dot{u}_{h}(0)=\mathrm{E} v_{0} .
$$

The set $X_{h}^{\circ}$ in (24c) is the polar set of $X_{h}$, i.e.,

$$
X_{h}^{\circ}:=\left\{\xi \in H^{1 / 2}(\Gamma):\left\langle\mu_{h}, \xi\right\rangle_{\Gamma}=0 \quad \forall \mu_{h} \in X_{h}\right\} .
$$

The Laplace operator $\Delta_{ \pm}$in (24a) is the one defined in the sense of distributions in $\mathbb{B} \backslash \Gamma$. Finally, the transmission condition (24d) is equivalent to

$$
\left\langle\llbracket \partial_{\nu} u_{h} \rrbracket, \xi_{h}\right\rangle_{\Gamma}=0 \quad \forall \xi_{h} \in X_{h}^{\circ}
$$

Let now $u_{h}$ be a solution of (24) $-(25)$ and define $\psi_{h}:=\llbracket \partial_{\nu} u_{h} \rrbracket=\llbracket \partial_{\nu}\left(u_{h}-u^{\text {free }}\right) \rrbracket($ recall (6) ). We can then show that $\mathcal{S} * \psi_{h}=u_{h}-u^{\text {free }}$ by comparing the transmission problems that both solutions satisfy. 
Formulation as a Cauchy problem. Consider the Hilbert spaces

$$
\begin{aligned}
H & :=L^{2}(\mathbb{B}), \\
V & :=\left\{u \in H_{0}^{1}(\mathbb{B}): \gamma u \in X_{h}^{\circ}\right\}, \\
D(A) & :=\left\{u \in V: \Delta_{ \pm} u \in L^{2}(\mathbb{B}), \quad \llbracket \partial_{\nu} u \rrbracket \in X_{h}\right\},
\end{aligned}
$$

endowed with the respective norms

$$
\|u\|_{H}:=\|u\|_{\mathbb{B}} \quad\|u\|_{V}:=\|\nabla u\|_{\mathbb{B}}, \quad\|u\|_{D(A)}:=\left(\|\nabla u\|_{\mathbb{B}}^{2}+\left\|\Delta_{ \pm} u\right\|_{\mathbb{B}}^{2}\right)^{1 / 2} .
$$

We also consider the operator $A:=\Delta_{ \pm}$. We next verify the two conditions of Section 8 , First of all, the generalized Green's Identity: for $u \in D(A), v \in V \subset H_{0}^{1}(\mathbb{B})$, using the weak definition of the normal derivatives, it follows that

$$
(\nabla u, \nabla v)_{\mathbb{B}}+\left(\Delta_{ \pm} u, v\right)_{\mathbb{B}}=\left\langle\llbracket \partial_{\nu} u \rrbracket, \gamma v\right\rangle_{\Gamma}=0,
$$

because $\llbracket \partial_{\nu} u \rrbracket \in X_{h}$ and $\gamma v \in X_{h}^{\circ}$. The second step is surjectivity: for any $f \in L^{2}(\mathbb{B})$ we want to find

$$
u \in D(A), \quad-\Delta u+u=f \quad \text { in } \mathbb{B} \backslash \Gamma .
$$

We first choose $u^{\mathrm{nh}} \in H_{0}^{1}(\mathbb{B})$ such that $-\Delta u^{\mathrm{nh}}+u^{\mathrm{nh}}=f$ in $\mathbb{B}$, and note that $u^{\mathrm{nh}} \in H^{2}(\mathbb{B})$ by a simple regularity argument. We next consider a variational problem in the space $\underline{X}_{h}:=X_{h} \times H^{-1 / 2}(\partial \mathbb{B}) \subset H^{-1 / 2}(\Xi)$ :

$$
\left[\begin{array}{l}
\lambda=\left(\lambda_{h}, \lambda_{\partial}\right) \in \underline{X}_{h}, \\
\langle\rho, \mathrm{V} \lambda\rangle_{\Xi}=-\left\langle\rho_{h}, \gamma u^{\mathrm{nh}}\right\rangle_{\Gamma} \quad \forall \rho=\left(\rho_{h}, \rho_{\partial}\right) \in \underline{X}_{h} .
\end{array}\right.
$$

This problem is uniquely solvable by the coercivity property (20). We finally take $u:=$ $u^{\mathrm{nh}}+\mathrm{S} \lambda$. It is clear that $u \in H^{1}(\mathbb{B})$. Testing (27) with elements $\left(0, \rho_{\partial}\right) \in\{0\} \times H^{-1 / 2}(\partial \mathbb{B})$ and recalling that $\gamma_{\Xi} \mathrm{S}=\mathrm{V}$ it follows that $u \in H_{0}^{1}(\mathbb{B})$. Testing with elements $\left(\rho_{h}, 0\right) \in$ $X_{h} \times\{0\}$ it follows that $\gamma u \in X_{h}^{\circ}$. This proves that $u \in V$. Also, $-\Delta u+u=f$ in $\mathbb{B} \backslash \Gamma$ and $\llbracket \partial_{\nu} u \rrbracket=\llbracket \partial_{\nu} \mathrm{S} \mu \rrbracket=\lambda_{h} \in X_{h}$. Therefore $u \in D(A)$ and (26) is satisfied.

Conclusions. The theory for abstract Cauchy problems that is sketched in Section 8 can be applied to problem (24). When initial data are in $D(A) \times V$, the Cauchy problem has a unique strong $\mathcal{C}^{2}$ solution. Since $u_{0} \in H^{2}\left(\Omega^{+}\right)$and $v_{0} \in H^{1}\left(\Omega^{+}\right)$vanish in a neighborhood of $\bar{\Omega}$ and also in a neighborhood of $\partial \mathbb{B}$, then $\left(\mathrm{E} u_{0}, \mathrm{E} v_{0}\right)$ satisfies the regularity requirements to have strong solutions:

$$
u_{h} \in \mathcal{C}^{2}\left([0, T] ; L^{2}(\mathbb{B})\right) \cap \mathcal{C}^{1}\left([0, T] ; H_{0}^{1}(\mathbb{B})\right) \cap \mathcal{C}([0, T] ; D(A)) .
$$

Extending by zero to the exterior of $\mathbb{B}$, it is clear that (15) is satisfied. Note that the property is proved in $[0, T]$ for any $T$ and that $T$ influences the size of the ball $\mathbb{B}$ that we use to truncate the domain. Note also that $\llbracket \partial_{\nu} \cdot \rrbracket: D(A) \rightarrow H^{-1 / 2}(\Gamma)$ is bounded. Then $\psi_{h}:=\llbracket \partial_{\nu} u_{h} \rrbracket \in \mathcal{C}\left([0, \infty) ; H^{-1 / 2}(\Gamma)\right)$.

Remark 3.1. The convenience of adding the cut-off boundary $\partial \mathbb{B}$-far enough from the obstacle so that the solution is not affected in the time interval $[0, T]$ - is due to the difficulty of working with energy spaces in unbounded domains (see Section 8). 


\section{Transparent boundary conditions}

We now consider the problem of propagation of compactly supported initial conditions in free space $\mathbb{R}^{d}$

$$
c^{-2} \ddot{u}=\nabla \cdot(\kappa \nabla u)
$$

with initial conditions

$$
u(0)=u_{0}, \quad \dot{u}(0)=v_{0} .
$$

We assume that $\kappa, c \in L^{\infty}\left(\mathbb{R}^{d}\right)$ are positive and that $\kappa^{-1}, c^{-1} \in L^{\infty}\left(\mathbb{R}^{d}\right)$. Furthermore, we assume that $c \equiv 1$ and $\kappa \equiv 1$ outside a compact set. Let

$$
\mathcal{O}:=\operatorname{supp} u_{0} \cup \operatorname{supp} v_{0} \cup \operatorname{supp}(c-1) \cup \operatorname{supp}(\kappa-1)
$$

which is a compact set by all the previous hypotheses on initial data and coefficients. We choose a bounded open set $\Omega$ with Lipschitz boundary $\Gamma:=\partial \Omega$, with connected exterior $\Omega^{+}:=\mathbb{R}^{d} \backslash \Gamma$ and such that $\mathcal{O} \subset \bar{\Omega}$. We admit the possibility of taking $\bar{\Omega}=\mathcal{O}$, as long as $\mathcal{O}$ meets the regularity hypotheses required for $\Omega$. Also, there is no need to have $\Omega$ connected, although for simplicity we will assume that its exterior is connected.

Denoting the conormal derivative on $\Gamma$ by $\partial_{\nu}^{\kappa} u=(\kappa \nabla u) \cdot \boldsymbol{\nu}$, and renaming $u^{\text {ext }}=\left.u\right|_{\Omega^{+}}$, we have the problem

$$
\begin{aligned}
c^{-2} \ddot{u}=\nabla \cdot(\kappa \nabla u) & \text { in } \Omega, \\
\ddot{u}^{\mathrm{ext}}=\Delta u^{\mathrm{ext}} & \text { in } \Omega^{+}, \\
\gamma u=\gamma^{+} u^{\mathrm{ext}} & \text { on } \Gamma, \\
\partial_{\nu}^{\kappa} u=\partial_{\nu} u^{\mathrm{ext}} & \text { on } \Gamma,
\end{aligned}
$$

with initial conditions

$$
u(0)=u_{0}, \quad \dot{u}(0)=v_{0}, \quad u^{\text {ext }}(0)=0, \quad \dot{u}^{\operatorname{ext}}(0)=0 .
$$

The function $u^{\text {ext }}$ can be represented with Kirchhoff's formula

$$
u^{\mathrm{ext}}=\mathcal{D} * \varphi-\mathcal{S} * \lambda \quad(\varphi, \lambda):=\left(\gamma^{+} u^{\mathrm{ext}}, \partial_{\nu}^{+} u^{\mathrm{ext}}\right)
$$

using both Cauchy data on the interface $\Gamma$. This formula employs the double layer retarded potential (see [4], [14])

$$
(\mathcal{D} * \varphi)(\mathbf{x}, t)=\left\{\begin{aligned}
\left.\int_{\Gamma} \nabla_{\mathbf{y}}\left(\frac{\varphi(\mathbf{z}, t-|\mathbf{x}-\mathbf{y}|)}{4 \pi|\mathbf{x}-\mathbf{y}|}\right)\right|_{\mathbf{z}=\mathbf{y}} \cdot \boldsymbol{\nu}(\mathbf{y}) \mathrm{d} \Gamma(\mathbf{y}) \quad & (\text { when } d=3) \\
\int_{\Gamma} \int_{0}^{t-|\mathbf{x}-\mathbf{y}|} \frac{\varphi(\mathbf{y}, \tau)}{|\mathbf{x}-\mathbf{y}|^{2}-(t-\tau)^{2}} \Phi(\mathbf{x}, \mathbf{y}, t-\tau) \mathrm{d} \Gamma(\mathbf{y}) \mathrm{d} \tau & \\
& +\int_{\Gamma} \frac{\varphi(\mathbf{y}, t-|\mathbf{x}-\mathbf{y}|)}{|\mathbf{x}-\mathbf{y}|} \Phi(\mathbf{x}, \mathbf{y}, t-\tau) \mathrm{d} \Gamma(\mathbf{y}) \quad(\text { when } d=2),
\end{aligned}\right.
$$

where

$$
\Phi(\mathbf{x}, \mathbf{y}, t)=\frac{(\mathbf{x}-\mathbf{y}) \cdot \boldsymbol{\nu}(\mathbf{y})}{\sqrt{t^{2}-|\mathbf{x}-\mathbf{y}|^{2}}}
$$


and $\boldsymbol{\nu}(\mathbf{y})$ is the outwards pointing normal vector at $\mathbf{y}$. Three retarded integral operators appear in this formulation:

$$
\mathcal{K}^{t} * \lambda:=\frac{1}{2}\left(\partial_{\nu}^{+}+\partial_{\nu}^{-}\right)(\mathcal{S} * \lambda) \quad \mathcal{K} * \varphi=\frac{1}{2}\left(\gamma^{+}+\gamma^{-}\right)(\mathcal{D} * \varphi) \quad \mathcal{W} * \varphi=-\partial_{\nu}^{ \pm}(\mathcal{D} * \varphi) .
$$

The boundary-field formulation is based on representing $u^{\text {ext }}$ with the formula (30). We then write a weak formulation for the interior equation (28a) and substitute the transmission condition (28d):

$$
\left(c^{-2} \ddot{u}, v\right)_{\Omega}+(\kappa \nabla u, \nabla v)_{\Omega}-\langle\lambda, \gamma v\rangle_{\Gamma}=0 \quad \forall v \in H^{1}(\Omega) .
$$

A second equation is obtained by imposing the transmission condition (28c), with $u^{\text {ext }}$ written in terms of its Cauchy data (30) and using the conditions (31) to represent the trace of $\mathcal{D} * \varphi$ :

$$
\gamma u+\mathcal{V} * \lambda-\left(\frac{1}{2} \varphi+\mathcal{K} * \varphi\right)=0
$$

The third equation is an identity satisfied by the Cauchy data:

$$
\frac{1}{2} \lambda+\mathcal{K}^{t} * \lambda+\mathcal{W} * \varphi=0
$$

Finally, the global formulation results from writing (32)-(33)-(34). The unknowns are

$$
u:[0, \infty) \rightarrow H^{1}(\Omega), \quad \lambda: \mathbb{R} \rightarrow H^{-1 / 2}(\Gamma), \quad \varphi: \mathbb{R} \rightarrow H^{1 / 2}(\Gamma),
$$

with initial conditions

$$
u(0)=u_{0}, \quad \dot{u}(0)=v_{0}, \quad \lambda \equiv 0 \text { in }(-\infty, 0), \quad \varphi \equiv 0 \text { in }(-\infty, 0) .
$$

We now choose spaces

$$
V_{h} \subset H^{1}(\Omega) \quad X_{h} \subset H^{-1 / 2}(\Gamma) \quad Y_{h} \subset H^{1 / 2}(\Gamma),
$$

and look for

$$
u_{h}:[0, \infty) \rightarrow V_{h}, \quad \lambda_{h}: \mathbb{R} \rightarrow X_{h}, \quad \varphi_{h}: \mathbb{R} \rightarrow Y_{h},
$$

satisfying initial conditions

$$
u_{h}(0)=u_{h, 0}, \quad \dot{u}_{h}(0)=v_{h, 0}, \quad \lambda_{h} \equiv 0 \text { in }(-\infty, 0), \quad \varphi_{h} \equiv 0 \text { in }(-\infty, 0),
$$

for approximations $u_{0} \approx u_{h, 0} \in V_{h}$ and $v_{0} \approx v_{h, 0} \in V_{h}$ to be determined by a projection method or by some kind of interpolation process (this is not relevant in the sequel). Finally, we have the set of Galerkin equations: for $t>0$

$$
\begin{array}{rlrl}
\left(c^{-2} \ddot{u}_{h}, v_{h}\right)_{\Omega}+\left(\kappa \nabla u_{h}, \nabla v_{h}\right)_{\Omega}-\left\langle\lambda_{h}, \gamma v_{h}\right\rangle_{\Gamma} & =0 & & \forall v_{h} \in V_{h}, \\
\left\langle\mu_{h}, \gamma u_{h}\right\rangle_{\Gamma}+\left\langle\mu_{h}, \mathcal{V} * \lambda_{h}\right\rangle_{\Gamma}-\left\langle\mu_{h}, \frac{1}{2} \varphi_{h}+\mathcal{K} * \varphi_{h}\right\rangle_{\Gamma}=0 & & \forall \mu_{h} \in X_{h}, \\
\left\langle\frac{1}{2} \lambda_{h}+\mathcal{K}^{t} * \lambda_{h}, \xi_{h}\right\rangle_{\Gamma}+\left\langle\mathcal{W} * \varphi_{h}, \xi_{h}\right\rangle_{\Gamma}=0 & & \forall \xi_{h} \in Y_{h} .
\end{array}
$$

Equations (36) form a system of linear second order differential equations coupled with the kind of retarded equations that we found in Section 3 (see (12) for instance). These equations are complemented with the initial conditions (35). Once (35)-(36) has been 
solved (its solvability is part of what we state in the next theorem), we can define the approximation to the exterior solution

$$
u_{h}^{\mathrm{ext}}:=\mathcal{D} * \varphi_{h}-\mathcal{S} * \lambda_{h}
$$

Note that this function is defined in $\mathbb{R}^{d}$ and not only in $\Omega^{+}$. In order to simplify some of the forthcoming arguments, we will assume that constant functions belong to the three discrete spaces

$$
\mathbb{P}_{0}(\Omega) \subset V_{h} \quad \mathbb{P}_{0}(\Gamma) \subset X_{h} \quad \mathbb{P}_{0}(\Gamma) \subset Y_{h}
$$

When $\Gamma$ and $\Omega$ are not connected, the spaces $\mathbb{P}_{0}(\Gamma)$ and $\mathbb{P}_{0}(\Omega)$ have to be understood as the spaces of constant functions on each connected component of the corresponding domain.

The following result (which will be proved in Section 5) gives a basic regularity estimate for the solution of this problem. It also states an energy conservation property, where in addition to the expected wave fields $\left(u_{h}\right.$ in the interior domain $\Omega$ and $u_{h}^{\text {ext }}$ in the exterior domain $\Omega^{+}$), we have to count the energy of $u_{h}^{\text {ext }}$ in the interior of $\Gamma$, computed with the material properties of the surrounding medium.

Theorem 4.1. The semidiscrete total fields $\left(u_{h}, u_{h}^{\mathrm{ext}}\right)$ and the approximations to the Cauchy data on the interface $\left(\varphi_{h}, \lambda_{h}\right)$ given by (35)-(36)-(37) satisfy

$$
\begin{aligned}
u_{h} & \in \mathcal{C}^{2}\left([0, \infty) ; L^{2}(\Omega)\right) \cap \mathcal{C}^{1}\left([0, \infty) ; H^{1}(\Omega)\right), \\
u_{h}^{\text {ext }} & \in \mathcal{C}^{2}\left([0, \infty) ; L^{2}\left(\mathbb{R}^{d}\right) \cap \mathcal{C}^{1}\left([0, \infty) ; H^{1}\left(\mathbb{R}^{d} \backslash \Gamma\right)\right),\right. \\
\lambda_{h} & \in \mathcal{C}\left([0, \infty) ; H^{-1 / 2}(\Gamma)\right), \\
\varphi_{h} & \in \mathcal{C}^{1}\left([0, \infty) ; H^{1 / 2}(\Gamma)\right) .
\end{aligned}
$$

Moreover, the energy

$$
\frac{1}{2}\left\|\kappa^{1 / 2} \nabla u_{h}(t)\right\|_{\Omega}^{2}+\frac{1}{2}\left\|\nabla u_{h}^{\text {ext }}(t)\right\|_{\mathbb{R}^{d} \backslash \Gamma}^{2}+\frac{1}{2}\left\|c^{-1} \dot{u}_{h}(t)\right\|_{\Omega}^{2}+\frac{1}{2}\left\|\dot{u}_{h}^{\text {ext }}(t)\right\|_{\mathbb{R}^{d}}
$$

is constant over time.

\section{$5 \quad$ Proof of Theorem 4.1}

Introduction of a cut-off boundary. We will prove the theorem for an arbitrary interval $[0, T]$. Since $u_{h}^{\text {ext }}$ is defined with retarded potentials whose densities are causal functions (see the initial conditions (35) ), we can pick a sufficiently large radius $R>0$ so that

$$
\operatorname{supp} u_{h}^{\operatorname{ext}}(t) \subset \mathbb{B}:=B(\mathbf{0} ; R) \quad \forall t \in[0, T] .
$$

A transmission problem. The first step towards the proof consists of writing (36) in terms of the fields $\left(u_{h}, u_{h}^{\text {ext }}\right)$. The field $u_{h}^{\text {ext }}$ satisfies the wave equation in $\mathbb{R}^{d} \backslash \Gamma$. The interior field $u_{h}$ does not satisfy a differential equation though. Note that:

$$
\llbracket \gamma u_{h}^{\mathrm{ext}} \rrbracket=-\varphi_{h} \quad \llbracket \partial_{\nu} u_{h}^{\mathrm{ext}} \rrbracket=-\lambda_{h}
$$


The transmission problem looks for

$$
u_{h}:[0, \infty) \rightarrow V_{h}, \quad u_{h}^{\text {ext }}:[0, \infty) \rightarrow H^{1}(\mathbb{B} \backslash \Gamma)
$$

satisfying initial conditions

$$
u_{h}(0)=u_{h, 0}, \quad \dot{u}_{h}(0)=v_{h, 0}, \quad u_{h}^{\operatorname{ext}}(0)=0, \quad \dot{u}_{h}^{\operatorname{ext}}(0)=0,
$$

and the equations for all $t>0$

$$
\begin{aligned}
& \left(c^{-2} \ddot{u}_{h}, v_{h}\right)_{\Omega}+\left(\kappa \nabla u_{h}, \nabla v_{h}\right)+\left\langle\llbracket \partial_{\nu} u_{h}^{\text {ext }} \rrbracket, \gamma v_{h}\right\rangle_{\Gamma}=0 \quad \forall v_{h} \in V_{h}, \\
& \ddot{u}_{h}^{\mathrm{ext}}=\Delta_{ \pm} u_{h}^{\mathrm{ext}}, \\
& \gamma_{\partial \mathbb{B}} u_{h}^{\text {ext }}=0, \\
& \llbracket \gamma u_{h}^{\text {ext }} \rrbracket \in Y_{h}, \quad \gamma u_{h}-\gamma^{+} u_{h}^{\text {ext }} \in X_{h}^{\circ}, \\
& \llbracket \partial_{\nu} u_{h}^{\mathrm{ext}} \rrbracket \in X_{h}, \quad \partial_{\nu}^{-} u_{h}^{\mathrm{ext}} \in Y_{h} .
\end{aligned}
$$

Equation (47a) corresponds to (36a) after substituting $\lambda_{h}=-\llbracket \partial_{\nu} u_{h}^{\text {ext }}$ (see (44). The exterior boundary condition (47b) is a consequence of (43). The first condition in both (47d) and (47e) is a consequence of (44). The second condition in (47d) is just (36b). Finally, the second condition in (47e) is (36c).

Formulation as a Cauchy problem. Consider the space

$$
H_{\partial \mathbb{B}}^{1}(\mathbb{B} \backslash \Gamma):=\left\{u \in H^{1}(\mathbb{B} \backslash \Gamma): \gamma_{\partial \mathbb{B}} u=0\right\} \cong H^{1}(\Omega) \times H_{\partial \mathbb{B}}^{1}\left(\mathbb{B} \cap \Omega^{+}\right)
$$

(recall (16) ). The three required spaces to fit in the frame of Section 8 are:

$$
\begin{aligned}
H & :=V_{h} \times L^{2}(\mathbb{B}) \\
V & :=\left\{\left(u_{h}, u^{\star}\right) \in V_{h} \times H_{\partial \mathbb{B}}^{1}(\mathbb{B} \backslash \Gamma): \llbracket \gamma u^{\star} \rrbracket \in Y_{h} \quad \gamma u_{h}-\gamma^{+} u^{\star} \in X_{h}^{\circ}\right\} \\
D(A) & :=\left\{\left(u_{h}, u^{\star}\right) \in V: \Delta_{ \pm} u^{\star} \in L^{2}(\mathbb{B}) \quad \llbracket \partial_{\nu} u^{\star} \rrbracket \in X_{h}, \quad \partial_{\nu} u^{\star} \in Y_{h}^{\circ}\right\} .
\end{aligned}
$$

The norm of $H$ is

$$
\left\|\left(u_{h}, u^{\star}\right)\right\|_{H}^{2}:=\left\|c^{-1} u_{h}\right\|_{\Omega}^{2}+\left\|u^{\star}\right\|_{\mathbb{B}}^{2} .
$$

The following norm

$H^{1}(\Omega) \times H_{\partial \mathbb{B}}^{1}(\mathbb{B} \backslash \Gamma) \ni\left(u, u^{\star}\right) \mapsto\left\|\kappa^{1 / 2} \nabla u\right\|_{\Omega}^{2}+\left\|\nabla u^{\star}\right\|_{\mathbb{B} \backslash \Gamma}^{2}+\left|\int_{\Gamma}\left(\gamma u-\gamma^{+} u\right)\right|^{2}+\left|\frac{1}{|\Omega|} \int_{\Omega} u^{\star}\right|^{2}$.

can be easily shown to be equivalent to the usual Sobolev norm in this space. This allows us to write a norm in $V$ :

$$
\left\|\left(u_{h}, u^{\star}\right)\right\|_{V}^{2}:=\left\|\kappa^{1 / 2} \nabla u_{h}\right\|_{\Omega}^{2}+\left\|\nabla u^{\star}\right\|_{\mathbb{B} \backslash \Gamma}^{2}+\left|\frac{1}{|\Omega|} \int_{\Omega} u^{\star}\right|^{2},
$$

since we have assumed that $\mathbb{P}_{0}(\Gamma) \subset X_{h}$. For $D(A)$ we define the norm

$$
\left\|\left(u_{h}, u^{\star}\right)\right\|_{D(A)}^{2}:=\left\|\left(u_{h}, u^{\star}\right)\right\|_{V}^{2}+\left\|\Delta_{ \pm} u^{\star}\right\|_{\mathbb{B} \backslash \Gamma}^{2} .
$$


To define the operator $A: D(A) \rightarrow H$ associated to the evolution problem (47), we introduce the operators $\Delta_{h}^{\kappa}: H^{1}(\Omega) \rightarrow V_{h}$ and $\gamma_{h}^{t}: H^{-1 / 2}(\Gamma) \rightarrow V_{h}$, defined by the discrete equations

$$
\left(c^{-2} \Delta_{h}^{\kappa} u, v_{h}\right)_{\Omega}=-\left(\kappa \nabla u, \nabla v_{h}\right)_{\Omega} \quad \forall v_{h} \in V_{h}
$$

and

$$
\left(c^{-2} \gamma_{h}^{t} \lambda, v_{h}\right)_{\Omega}=\left\langle\lambda, \gamma v_{h}\right\rangle_{\Gamma} \quad \forall v_{h} \in V_{h},
$$

respectively. These operators are defined so that (47a) can be rewritten as $\ddot{u}_{h}=\Delta_{h}^{\kappa} u_{h}-$ $\gamma_{h}^{t} \llbracket \partial_{\nu} u_{h}^{\text {ext }} \rrbracket$. The operator $A: D(A) \rightarrow H$ is then defined by

$$
A\left(u_{h}, u^{\star}\right):=\left(\Delta_{h}^{\kappa} u_{h}-\gamma_{h}^{t} \llbracket \partial_{\nu} u^{\star} \rrbracket, \Delta_{ \pm} u^{\star}\right)
$$

Problem (47) has the general form (87) with initial data $\left(u_{h, 0}, 0\right) \in D(A)$ and $\left(v_{h, 0}, 0\right) \in V$.

Rigid motions of the system. Let $M:=\{0\} \times \operatorname{span}\left\{\chi_{\Omega}\right\}$. Noting that

$$
\llbracket \partial_{\nu} \chi_{\Omega} \rrbracket=0, \quad \llbracket \gamma_{\Omega} \rrbracket=1 \in Y_{h}, \quad \gamma_{\partial \mathbb{B}} \chi_{\Omega}=0, \quad \partial_{\nu}^{-} \chi_{\Omega}=0, \quad \text { and } \quad \gamma^{+} \chi_{\Omega}=0,
$$

it is simple to check that $M \subset D(A)$ and $M \subset \operatorname{ker}(A)$. We consider the following seminorm in $V$

$$
\left|\left(u_{h}, u^{\star}\right)\right|_{V}^{2}:=\left\|\kappa^{1 / 2} \nabla u_{h}\right\|_{\Omega}^{2}+\left\|\nabla u^{\star}\right\|_{\mathbb{B} \backslash \Gamma}^{2},
$$

associated to a semi-inner product $\left[\left(u_{h}, u^{\star}\right),\left(v_{h}, v^{\star}\right)\right]_{V}$. The hypotheses to consider the finite dimensional space $M$ as a space of rigid motions of the evolution problem (87) (see Section 8) are then easily verified.

Verification of the associated Green's Identity. Let $\underline{u}:=\left(u_{h}, u^{\star}\right) \in D(A)$ and $\underline{v}:=$ $\left(v_{h}, v^{\star}\right) \in V$. Using the definition of the discrete operators (51)-(52) and the definition of the weak normal derivatives, it follows that

$$
\begin{aligned}
(A \underline{u}, \underline{v})_{H}+[\underline{u}, \underline{v}]_{V}= & \left(c^{-2} \Delta_{h}^{\kappa} u_{h}, v_{h}\right)_{\Omega}-\left(c^{-2} \gamma_{h}^{t} \llbracket \partial_{\nu} u^{\star} \rrbracket, v_{h}\right)_{\Omega}+\left(\Delta_{ \pm} u^{\star}, v^{\star}\right)_{\mathbb{B} \backslash \Gamma} \\
& +\left(\kappa \nabla u_{h}, \nabla v_{h}\right)_{\Omega}+\left(\nabla u^{\star}, \nabla v^{\star}\right)_{\mathbb{B} \backslash \Gamma} \\
= & -\left\langle\llbracket \partial_{\nu} u^{\star} \rrbracket, \gamma v_{h}\right\rangle_{\Gamma}+\left\langle\partial_{\nu}^{-} u^{\star}, \gamma^{-} v^{\star}\right\rangle_{\Gamma}-\left\langle\partial_{\nu}^{+} u^{\star}, \gamma^{+} v^{\star}\right\rangle_{\Gamma} \\
= & -\langle\underbrace{\left\langle\llbracket \partial_{\nu} u^{\star} \rrbracket\right.}_{\in X_{h}}, \underbrace{\gamma v_{h}-\gamma^{+} v^{\star}}_{\in X_{h}^{\circ}}\rangle_{\Gamma}-\langle\underbrace{\partial_{\nu}^{-} u^{\star}}_{\in Y_{h}^{\circ}}, \underbrace{\llbracket \gamma v^{\star} \rrbracket}_{\in Y_{h}}\rangle_{\Gamma}=0 .
\end{aligned}
$$

The Yukawa double layer potential. For the proof of the surjectivity we need to introduce the double layer potential for the Yukawa operator on $\Xi=\Gamma \cup \partial \mathbb{B}$

$$
\mathrm{D} \varphi:=\int_{\Xi} \nabla_{\mathbf{y}} E(\cdot, \mathbf{y}) \varphi(\mathbf{y}) \mathrm{d} \Xi(\mathbf{y})
$$

where $E$ is given in (17). This potential defines a bounded operator

$$
\mathrm{D}: H^{1 / 2}(\Xi) \rightarrow H^{1}\left(\mathbb{R}^{d} \backslash \Xi\right)
$$


such that $\Delta(\mathrm{D} \varphi)=\mathrm{D} \varphi$ in $\mathbb{R}^{d} \backslash \Xi$ for all $\varphi$. Two bounded integral operators are associated to this potential

$$
\mathrm{K}:=\frac{1}{2}\left(\gamma_{\Xi}^{+}+\gamma_{\Xi}^{-}\right) \mathrm{D}: H^{1 / 2}(\Xi) \rightarrow H^{1 / 2}(\Xi), \quad \mathrm{W}:=-\partial_{\nu, \Xi}^{ \pm} \mathrm{D}: H^{1 / 2}(\Xi) \rightarrow H^{-1 / 2}(\Xi) .
$$

The operator $\mathrm{W}$ is coercive [22]

$$
\langle\mathrm{W} \varphi, \varphi\rangle_{\Xi} \geq C\|\varphi\|_{1 / 2, \Xi}^{2} \quad \forall \varphi \in H^{1 / 2}(\Xi) .
$$

Finally the adjoint of $\mathrm{K}$ satisfies $\mathrm{K}^{t}=\frac{1}{2}\left(\partial_{\nu, \Xi}^{+}+\partial_{\nu, \Xi}^{-}\right) \mathrm{S}$, where $\mathrm{S}$ is the single layer potential defined in (18).

Verification of the surjectivity property. Let $\left(f_{h}, f\right) \in V_{h} \times L^{2}(\mathbb{B})=H$. As we did in Section 3 , we start by finding $u^{\mathrm{nh}} \in H^{2}(\mathbb{B}) \cap H_{0}^{1}(\mathbb{B})$ such that $-\Delta u^{\mathrm{nh}}+u^{\mathrm{nh}}=f$ in $\mathbb{B}$. We then look for

$$
\begin{aligned}
u_{h} \in V_{h}, \quad \lambda=\left(\lambda_{h}, \lambda_{\partial}\right) \in \underline{X}_{h}:=X_{h} \times H^{-1 / 2}(\partial \mathbb{B}) \subset H^{-1 / 2}(\Xi) \\
\varphi=\left(\varphi_{h}, 0\right) \in \underline{Y}_{h}:=Y_{h} \times\{0\} \subset H^{1 / 2}(\Xi)
\end{aligned}
$$

satisfying the equations:

$$
\begin{aligned}
\left(c^{-2} u_{h}, v_{h}\right)_{\Omega}+\left(\kappa \nabla u_{h}, \nabla v_{h}\right)_{\Omega}-\left\langle\lambda_{h}, \gamma v_{h}\right\rangle_{\Gamma} & =\left(c^{-2} f_{h}, v_{h}\right)_{\Omega} & & \forall v_{h} \in V_{h}, \\
\left\langle\mu_{h}, \gamma u_{h}\right\rangle_{\Gamma}+\langle\mu, \mathrm{V} \lambda\rangle_{\Xi}-\left\langle\mu, \frac{1}{2} \varphi+\mathrm{K} \varphi\right\rangle_{\Xi} & =\left\langle\mu_{h}, \gamma u^{\mathrm{nh}}\right\rangle_{\Gamma} & & \forall \mu=\left(\mu_{h}, \mu_{\partial}\right) \in \underline{X}_{h} \\
\left\langle\frac{1}{2} \lambda+\mathrm{K}^{t} \lambda, \xi\right\rangle_{\Xi}+\langle\mathrm{W} \varphi, \xi\rangle_{\Xi} & =\left\langle\partial_{\nu} u^{\mathrm{nh}}, \xi_{h}\right\rangle_{\Gamma} & & \forall \xi=\left(\xi_{h}, 0\right) \in \underline{Y}_{h}
\end{aligned}
$$

Note that because of the particular form of the space $\underline{Y}_{h}$, the three bilinear forms where either $\xi$ or $\varphi$ appear are actually duality products in $\Gamma$. The bilinear form of problem (56) is coercive in $H^{1}(\Omega) \times H^{-1 / 2}(\Xi) \times H^{1 / 2}(\Xi)$ by (20) and (55). Therefore problem (56) has a unique solution. The final step is the verification that the pair

$$
\left(u_{h}, u^{\star}\right):=\left(u_{h}, u^{\mathrm{nh}}+\mathrm{D} \varphi-\mathrm{S} \lambda\right)
$$

belongs to $D(A)$ and that $(I-A)\left(u_{h}, u^{\star}\right)=\left(f_{h}, f\right)$. This follows from several simple arguments that we next list. Because of the potential form for $u^{\star}$ and the smoothness of $u^{\mathrm{nh}}$ across $\Gamma$ it follows that

$$
\llbracket \partial_{\nu} u^{\star} \rrbracket=-\lambda_{h} \in X_{h}, \quad \llbracket \gamma u^{\star} \rrbracket=-\varphi_{h} \in Y_{h} .
$$

Also

$$
\Delta_{ \pm} u^{\star}-u^{\star}=f
$$

and therefore $\Delta_{ \pm} u^{\star} \in L^{2}(\mathbb{B})$, while $u^{\star} \in H^{1}(\mathbb{B} \backslash \Gamma)$ because $u^{\text {nh }} \in H^{2}(\mathbb{B})$ and the mapping properties of potentials (19), (54) hold. Substituting (57) in (56a) and using the definitions of the discrete operators (51)-(52) it follows that

$$
u_{h}-\Delta_{h}^{\kappa} u_{h}+\gamma_{h}^{t} \llbracket \partial_{\nu} u^{\star} \rrbracket=f_{h} .
$$


If we test (56b) with elements $\left(0, \mu_{\partial}\right) \in\{0\} \times H^{1 / 2}(\partial \mathbb{B})$ it follows that $\gamma_{\partial \mathbb{B}} u^{\star}=0$. If we test with $\left(\mu_{h}, 0\right) \in X_{h} \times\{0\}$, it follows that

$$
\gamma u_{h}-\gamma^{+} u^{\star}=\gamma u_{h}-\mathrm{V} \lambda-\left(\frac{1}{2} \varphi_{h}+\mathrm{K} \varphi\right)+\gamma u^{\mathrm{nh}} \in X_{h}^{\circ}
$$

Finally, equation (56c) is equivalent to asserting that

$$
\partial_{\nu}^{+} u^{\star}=\partial_{\nu} u^{\mathrm{nh}}-\frac{1}{2} \lambda_{h}+\mathrm{K}^{t} \lambda-\mathrm{W} \varphi \in Y_{h}^{\circ}
$$

The preceding arguments have shown that $\left(u_{h}, u^{\star}\right) \in D(A)$ while (58)-(59) proves that $(I-A)\left(u_{h}, u^{\star}\right)=\left(f_{h}, f\right)$.

Conclusion. We can now apply the theory for Cauchy problems exposed in Section 8 , The statements of Theorem 4.1 are a direct consequence of these results.

\section{Two simple extensions}

We now show two other situations where the techniques developed in the preceding sections can be applied with minor modifications.

\subsection{Double layer potentials for sound-hard scattering}

Let us consider again the geometrical setting of Section 2. The problem of sound-hard scattering by an obstacle occupying the region $\bar{\Omega}$ can be expressed with the equations (1) -(2) with the boundary condition (1b) substituted by $\partial_{\nu} u=0$ on $\Gamma$ for all $t$. The assumptions on the initial data are the same as those given in Section 2, If we define $u^{\text {free }}$ with (4)-(5), the solution to this problem can be expressed as $u=\mathcal{D} * \varphi+u^{\text {free }}$, where $\varphi: \mathbb{R} \rightarrow H^{1 / 2}(\Gamma)$ vanishes identically for negative values of $t$.

The semidiscrete formulation follows from choosing a discrete space

$$
\mathbb{P}_{0}(\Gamma) \subset Y_{h} \subset H^{1 / 2}(\Gamma)
$$

discretizing the boundary condition

$$
\left[\begin{array}{l}
\varphi_{h}: \mathbb{R} \rightarrow Y_{h}, \quad \varphi_{h} \equiv 0 \text { in }(-\infty, 0) \\
\left\langle-\mathcal{W} * \varphi_{h}+\partial_{\nu} u^{\text {free }}, \xi_{h}\right\rangle_{\Gamma}=0 \quad \forall \xi_{h} \in Y_{h} \quad \forall t
\end{array}\right.
$$

and proposing

$$
u_{h}=\mathcal{D} * \varphi_{h}+u^{\text {free }}
$$

as approximation of $u$. Note that the following transmission conditions are satisfied for all $t \geq 0$ :

$$
\llbracket \gamma u_{h} \rrbracket \in Y_{h}, \quad \llbracket \partial_{\nu} u_{h} \rrbracket=0, \quad \partial_{\nu} u_{h} \in Y_{h}^{\circ}
$$


Theorem 6.1. The semidiscrete total field $u_{h}$ and the associated density $\varphi_{h}$ given by (60) and (61) satisfy

$$
\begin{aligned}
u_{h} & \in \mathcal{C}^{2}\left([0, \infty) ; L^{2}\left(\mathbb{R}^{d}\right)\right) \cap \mathcal{C}^{1}\left([0, \infty) ; H^{1}\left(\mathbb{R}^{d} \backslash \Gamma\right)\right) \\
\varphi_{h} & \in \mathcal{C}^{1}\left([0, \infty) ; H^{1 / 2}(\Gamma)\right)
\end{aligned}
$$

Moreover, the energy

$$
\frac{1}{2}\left\|\nabla u_{h}(t)\right\|_{\mathbb{R}^{d} \backslash \Gamma}^{2}+\frac{1}{2}\left\|\dot{u}_{h}(t)\right\|_{\mathbb{R}^{d}}^{2}
$$

is constant over time.

Proof. The techniques for the proof of this result are very similar to those of Section 4. We will simply sketch the main steps. First of all we pick a fixed time interval $[0, T]$ and choose a ball $\mathbb{B}$ that contains the support of the solution for all $t \in[0, T]$. It is now more convenient to include a Neumann boundary condition on the cut-off boundary $\partial_{\nu, \partial \mathbb{B}} u_{h}=0$ for all $t$. The spaces for the formulation as a Cauchy problem are the following:

$$
\begin{aligned}
H & :=L^{2}(\mathbb{B}), \\
V & :=\left\{u \in H^{1}(\mathbb{B} \backslash \Gamma): \llbracket \gamma u \rrbracket \in Y_{h}\right\}, \\
D(A) & :=\left\{u \in V: \Delta_{ \pm} u \in L^{2}(\mathbb{B}), \quad \llbracket \partial_{\nu} u \rrbracket=0, \quad \partial_{\nu} u \in Y_{h}^{\circ}, \quad \partial_{\nu, \partial \mathbb{B}} u=0\right\} .
\end{aligned}
$$

The relevant norms and seminorms are:

$$
\|u\|_{H}:=\|u\|_{\mathbb{B}}, \quad|u|_{V}:=\|\nabla u\|_{\mathbb{B} \backslash \Gamma}, \quad\|u\|_{V}^{2}:=|u|_{V}^{2}+\left|\frac{1}{|\Omega|} \int_{\Omega} u\right|^{2}+\left|\frac{1}{\left|\mathbb{B} \cap \Omega^{+}\right|} \int_{\mathbb{B} \cap \Omega^{+}} u\right|^{2} .
$$

The associated operator is the same as in Section 4, namely $A:=\Delta_{ \pm}$. The space of associated rigid motions is two-dimensional $M:=\operatorname{span}\left\{\chi_{\Omega}, \chi_{\mathbb{B} \cap \Omega^{+}}\right\}$. The proof of the corresponding Green's Identity is straightforward. For surjectivity we proceed in two steps. Given $f \in L^{2}(\mathbb{B})$, we first choose $u^{\mathrm{nh}} \in H^{2}(\mathbb{B})$ such that $-\Delta u^{\mathrm{nh}}+u^{\mathrm{nh}}=f$ in $\mathbb{B}$ and $\partial_{\nu, \partial \mathbb{B}} u^{\mathrm{nh}}=0$. (Note that this is possible by basic regularity theorems of elliptic problems on smooth domains.) Next we look for $\varphi=\left(\varphi_{h}, \varphi_{\partial}\right) \in \underline{Y}_{h}:=Y_{h} \times H^{1 / 2}(\partial \mathbb{B}) \subset H^{1 / 2}(\Xi)$ that solves the coercive variational problem

$$
\left[\begin{array}{l}
\varphi \in \underline{Y}_{h}, \\
\langle\mathrm{~W} \varphi, \xi\rangle_{\Gamma}=\left\langle\partial_{\nu} u^{\mathrm{nh}}, \xi_{h}\right\rangle_{\Gamma}, \quad \forall \xi=\left(\xi_{h}, \xi_{\partial}\right) \in \underline{Y}_{h},
\end{array}\right.
$$

and define $u=u^{\mathrm{nh}}+\mathrm{D} \varphi$. (Notations for Yukawa potentials and operators are those of Section 5.) It is simple to prove that $u \in D(A)$ and $u-A u=f$, which finishes the proof.

\subsection{A tighter transparent boundary condition}

We now consider the transmission problem (28)-(29) of Section 4. Instead of the transparent boundary condition that uses approximations of both Cauchy data on $\Gamma$ it is possible to construct another boundary-field formulation in the spirit of the BEM-FEM coupling schemes of Martin Costabel [9] and Houde Han [15]. The boundary unknown 
is $\lambda:=\partial_{\nu} u^{\text {ext }}$. The identities of Cauchy data on the boundary and the transmission conditions (28c $)-(28 \mathrm{~d})$ can be used to produce the following equations:

$$
\frac{1}{2} \gamma u-\mathcal{K} * \gamma u+\mathcal{V} * \lambda=0 \quad-\partial_{\nu}^{\kappa} u=\mathcal{W} * \gamma u-\frac{1}{2} \lambda+\mathcal{K}^{t} * \lambda .
$$

In (68) and all similar future expressions, it will be understood that $\gamma u(t) \equiv 0$ for $t<0$, although $u(t)$ will be defined for $t \geq 0$ and we will not look for a smooth continuation of $u$ for negative values of $t$.

The boundary-field formulation looks for $u:[0, \infty) \rightarrow H^{1}(\Omega)$ and $\lambda: \mathbb{R} \rightarrow H^{-1 / 2}(\Gamma)$ satisfying initial conditions

$$
u(0)=u_{0}, \quad \dot{u}(0)=v_{0}, \quad \lambda \equiv 0 \text { in }(-\infty, 0),
$$

and the equations

$$
\begin{aligned}
\left(c^{-2} \ddot{u}, v\right)_{\Omega}+(\kappa \nabla u, \nabla v)_{\Omega}+\langle\mathcal{W} * \gamma u, \gamma v\rangle_{\Gamma} & \\
-\left\langle\frac{1}{2} \lambda-\mathcal{K}^{t} * \lambda, \gamma v\right\rangle_{\Gamma}=0 \quad & \forall v \in H^{1}(\Omega), \\
\left\langle\mu, \frac{1}{2} \gamma u-\mathcal{K} * \gamma u\right\rangle_{\Gamma}+\langle\mu, \mathcal{V} * \lambda\rangle_{\Gamma}=0 \quad & \forall \mu \in H^{-1 / 2}(\Gamma),
\end{aligned}
$$

for all $t$. The discrete version of these equations uses two spaces

$$
\mathbb{P}_{0}(\Omega) \subset V_{h} \subset H^{1}(\Omega), \quad \mathbb{P}_{0}(\Gamma) \subset X_{h} \subset H^{-1 / 2}(\Gamma),
$$

and looks for $u_{h}:[0, \infty) \rightarrow V_{h}$ and $\lambda_{h}: \mathbb{R} \rightarrow X_{h}$ such that

$$
u_{h}(0)=u_{h, 0}, \quad \dot{u}_{h}(0)=v_{h, 0}, \quad \lambda_{h} \equiv 0 \text { in }(-\infty, 0),
$$

and for all $t$ :

$$
\begin{aligned}
&\left(c^{-2} \ddot{u}_{h}, v_{h}\right)_{\Omega}+\left(\kappa \nabla u_{h}, \nabla v_{h}\right)_{\Omega}+\left\langle\mathcal{W} * \gamma u_{h}, \gamma v_{h}\right\rangle_{\Gamma} \\
&-\left\langle\frac{1}{2} \lambda_{h}-\mathcal{K}^{t} * \lambda_{h}, \gamma v_{h}\right\rangle_{\Gamma}=0 \quad \forall v_{h} \in V_{h}, \\
&\left\langle\mu_{h}, \frac{1}{2} \gamma u_{h}-\mathcal{K} * \gamma u_{h}\right\rangle_{\Gamma}+\left\langle\mu_{h}, \mathcal{V} * \lambda_{h}\right\rangle_{\Gamma}=0 \quad \forall \mu_{h} \in X_{h} .
\end{aligned}
$$

Compared with the semidiscrete system (36), this system has one less group of equations and unknowns. The price to pay is the fact that $u_{h}$ is affected by integral delay operators. The reconstructed exterior solution is given by

$$
u_{h}^{\text {ext }}:=\mathcal{D} * \gamma u_{h}-\mathcal{S} * \lambda_{h} .
$$

As in Section 4, we have to consider $u_{h}^{\text {ext }}$ defined in $\mathbb{R}^{d}$ (and not only in $\Omega^{+}$) to have the correct balance of energy.

Theorem 6.2. The semidiscrete total fields $\left(u_{h}, u_{h}^{\mathrm{ext}}\right)$ and the approximation to the normal derivative on the interface $\lambda_{h}$ given by (70)-(171)-(172) satisfy

$$
\begin{aligned}
u_{h} & \in \mathcal{C}^{2}\left([0, \infty) ; L^{2}(\Omega)\right) \cap \mathcal{C}^{1}\left([0, \infty) ; H^{1}(\Omega)\right), \\
u_{h}^{\text {ext }} & \in \mathcal{C}^{2}\left([0, \infty) ; L^{2}\left(\mathbb{R}^{d}\right) \cap \mathcal{C}^{1}\left([0, \infty) ; H^{1}\left(\mathbb{R}^{d} \backslash \Gamma\right)\right),\right. \\
\lambda_{h} & \in \mathcal{C}\left([0, \infty) ; H^{-1 / 2}(\Gamma)\right) .
\end{aligned}
$$

Moreover, the energy

$$
\frac{1}{2}\left\|\kappa^{1 / 2} \nabla u_{h}(t)\right\|_{\Omega}^{2}+\frac{1}{2}\left\|\nabla u_{h}^{\text {ext }}(t)\right\|_{\mathbb{R}^{d} \backslash \Gamma}^{2}+\frac{1}{2}\left\|c^{-1} \dot{u}_{h}(t)\right\|_{\Omega}^{2}+\frac{1}{2}\left\|\dot{u}_{h}^{\text {ext }}(t)\right\|_{\mathbb{R}^{d} \backslash \Gamma}
$$

is constant over time. 
Proof. The proof has a similar structure as that of Theorem 4.1. We will just point out the milestones of the proof. Choosing a ball $\mathbb{B}$ that contains the support of the solution for all $t \in[0, T]$ and adding a Dirichlet boundary condition on $\partial \mathbb{B}$, we can consider a Cauchy problem satisfied by the pair $\left(u_{h}, u_{h}^{\text {ext }}\right)$ with the following elements: the spaces

$$
\begin{aligned}
H & :=V_{h} \times L^{2}(\mathbb{B}), \\
V & :=\left\{\left(u_{h}, u^{\star}\right) \in H: \llbracket \gamma u^{\star} \rrbracket+\gamma u_{h}=0, \quad \gamma^{-} u^{\star} \in X_{h}^{\circ}, \quad \gamma_{\partial \mathbb{B}} u^{\star}=0\right\}, \\
D(A) & :=\left\{\left(u_{h}, u^{\star}\right) \in V: \Delta_{ \pm} u^{\star} \in L^{2}(\mathbb{B}), \quad \llbracket \partial_{\nu} u^{\star} \rrbracket \in X_{h}\right\},
\end{aligned}
$$

the norms

$$
\left\|\left(u_{h}, u^{\star}\right)\right\|_{H}^{2}:=\left\|c^{-1} u_{h}\right\|_{\Omega}^{2}+\left\|u^{\star}\right\|_{\mathbb{B}}^{2} \quad\left\|\left(u_{h}, u^{\star}\right)\right\|_{V}^{2}:=\left\|\kappa^{1 / 2} \nabla u_{h}\right\|_{\Omega}^{2}+\left\|\nabla u^{\star}\right\|_{\mathbb{B} \backslash \Gamma}^{2}
$$

and the operator

$$
A\left(u_{h}, u^{\star}\right):=\left(\Delta_{h}^{\kappa} u_{h}+\gamma_{h}^{t} \partial_{\nu}^{+} u^{\star}, \Delta_{ \pm} u^{\star}\right)
$$

(see (51) and (52)). The associated Green's Identity is straightforward to prove. To show surjectivity of $I-A$, we proceed as follows. Given $\left(f_{h}, f\right) \in H$, we first take $u^{\mathrm{nh}} \in H^{2}(\mathbb{B}) \cap H_{0}^{1}(\mathbb{B})$ satisfying $-\Delta u^{\mathrm{nh}}+u^{\mathrm{nh}}=f$ in $\mathbb{B}$. We then construct

$$
\left(u_{h}, u^{\star}\right)=\left(u_{h}, u^{\mathrm{nh}}+\mathrm{D} \widetilde{\gamma u_{h}}-\mathrm{S} \lambda\right)
$$

where

$$
u_{h} \in V_{h}, \quad \lambda=\left(\lambda_{h}, \lambda_{\partial}\right) \in \underline{X}_{h}:=X_{h} \times H^{-1 / 2}(\partial \mathbb{B}) \subset H^{-1 / 2}(\Xi)
$$

is the solution of

$$
\begin{aligned}
\left(c^{-2} u_{h}, v_{h}\right)_{\Omega}+\left(\kappa \nabla u_{h}, \nabla v_{h}\right)_{\Omega} & \\
+\left\langle\mathrm{W} \widetilde{\gamma u_{h}}, \widetilde{\gamma v_{h}}\right\rangle_{\Xi}-\left\langle\frac{1}{2} \lambda-\mathrm{K}^{t} \lambda, \widetilde{\gamma v_{h}}\right\rangle_{\Xi} & =\left(c^{-2} f_{h}, v_{h}\right)_{\Omega}+\left\langle\partial_{\nu} u^{\mathrm{nh}}, \gamma v_{h}\right\rangle_{\Gamma} \quad \forall v_{h} \in V_{h}, \\
\left\langle\mu, \frac{1}{2} \widetilde{\gamma u_{h}}-\mathrm{K} \widetilde{\gamma u_{h}}\right\rangle_{\Xi}+\langle\mu, \mathrm{V} \lambda\rangle_{\Xi} & =\left\langle\mu_{h}, \gamma u^{\mathrm{nh}}\right\rangle_{\Gamma} \quad \forall \mu=\left(\mu_{h}, \mu_{\partial}\right) \in \underline{X}_{h},
\end{aligned}
$$

and $\widetilde{\gamma u_{h}}=\left(\gamma u_{h}, 0\right) \in H^{1 / 2}(\Gamma) \times\{0\} \subset H^{1 / 2}(\Xi)$. Note that problem (78) is associated to a coercive bilinear form and is therefore uniquely solvable. It is then simple to prove that $\left(u_{h}, u^{\star}\right) \in D(A)$ and $A\left(u_{h}, u^{\star}\right)=\left(f_{h}, f\right)$, which finishes the proof.

\section{Two negative results}

This last section shows two results where Galerkin discretizations lead to problems with non-constant energy. This fact does not mean that the discretizations are not valid (they can still be stable), but at least shows how delicate the energy balance is when discretized integral operators are used.

\subsection{A direct method for sound-soft scattering}

Let us consider again the problem of Section 2. The solution of (1)-(2) can be decomposed using the free wave (solution to (44)-(5)) and Kirchhoff's formula for the scattered wave:

$$
u=u^{\text {free }}-\mathcal{D} * \gamma u^{\text {free }}-\mathcal{S} * \lambda, \quad \lambda:=\partial_{\nu}^{+} u-\partial_{\nu} u^{\text {free }},
$$


where $\lambda: \mathbb{R} \rightarrow H^{-1 / 2}(\Gamma)$ is causal and in the potential expression $\mathcal{D} * \gamma u^{\text {free }}$ we have to understand that $\gamma u^{\text {free }}: \mathbb{R} \rightarrow H^{1 / 2}(\Gamma)$ is a causal function even if $u^{\text {free }}$ is only defined for positive values of $t$. The indirect decomposition (9) used $u=\mathcal{S} * \psi+u^{\text {free }}$ was naturally extended to $\mathbb{R}^{d}$, with the result that $u(t) \equiv 0$ in $\Omega$ for all $t$. The extension to the interior domain of (79) is

$$
u=u^{\text {free }} \chi_{\Omega^{+}}-\mathcal{D} * \gamma u^{\text {free }}-\mathcal{S} * \lambda,
$$

because the potential expression $\mathcal{D} * \gamma u^{\text {free }}+\mathcal{S} * \lambda$ vanishes identically in $\Omega$ by Kirchhoff's formula. The discrete version of this process computes

$$
\left[\begin{array}{l}
\lambda_{h}: \mathbb{R} \rightarrow X_{h} \quad \lambda_{h} \equiv 0 \text { in }(-\infty, 0), \\
\left\langle\mu_{h}, \mathcal{V} * \lambda_{h}\right\rangle_{\Gamma}=\left\langle\mu_{h}, \frac{1}{2} \gamma u^{\text {free }}-\mathcal{K} * \gamma u^{\text {free }}\right\rangle_{\Gamma} \quad \forall \mu_{h} \in X_{h}, \quad \forall t,
\end{array}\right.
$$

and then constructs the total field

$$
u_{h}:=u^{\text {free }} \chi_{\Omega^{+}}-\mathcal{D} * \gamma u^{\text {free }}-\mathcal{S} * \lambda_{h} .
$$

Note that $u_{h}$ satisfies the non-homogeneous transmission problem

$$
\begin{array}{r}
\ddot{u}_{h}=\Delta_{ \pm} u_{h}, \\
\llbracket \gamma u_{h} \rrbracket=0, \\
\gamma u_{h} \in X_{h}^{\circ}, \\
\llbracket \partial_{\nu} u_{h} \rrbracket+\partial_{\nu} u^{\text {free }} \in X_{h} .
\end{array}
$$

(Compare with (24) and note that the condition at $\partial \mathbb{B}$ can always be added for finite time intervals.) The fact that this problem is a non-homogeneous version of a problem that is conservative gives a first hint that the natural energy of this problem will not be constant. Also, applying integration by parts and (82), we can prove that

$$
\frac{\mathrm{d}}{\mathrm{d} t}\left(\frac{1}{2}\left\|\dot{u}_{h}\right\|_{\mathbb{R}^{d}}^{2}+\frac{1}{2}\left\|\nabla u_{h}\right\|_{\mathbb{R}^{d} \backslash \Gamma}^{2}\right)=-\left\langle\partial_{\nu} u^{\text {free }}, \gamma \dot{u}_{h}\right\rangle_{\Gamma} .
$$

This shows that energy is not constant.

\subsection{Transparent conditions with one equation}

Consider again the propagation problem in free space (28). Instead of using two integral identities as in Sections 4 we can work with a single integral equation in the spirit of the one-equation coupling of BEM-FEM of Claus Johnson and Jean-Claude Nédélec [17, 23]. After space Galerkin discretization, the coupled system becomes an evolution problem that looks for $u_{h}:[0, \infty) \rightarrow V_{h}$ and $\lambda_{h}: \mathbb{R} \rightarrow X_{h}$ such that

$$
u_{h}(0)=u_{h, 0}, \quad \dot{u}_{h}(0)=v_{h, 0}, \quad \lambda_{h} \equiv 0 \text { in }(-\infty, 0),
$$

and for all $t \geq 0$

$$
\begin{aligned}
\left(c^{-2} \ddot{u}_{h}, v_{h}\right)_{\Omega}+\left(\kappa \nabla u_{h}, \nabla v_{h}\right)-\left\langle\lambda_{h}, \gamma v_{h}\right\rangle_{\Gamma} & =0 & & \forall v_{h} \in V_{h}, \\
\left\langle\mu_{h}, \frac{1}{2} \gamma u_{h}-\mathcal{K} * \gamma u_{h}\right\rangle_{\Gamma}+\left\langle\mu_{h}, \mathcal{V} * \lambda_{h}\right\rangle_{\Gamma} & =0 & & \forall \mu_{h} \in X_{h}
\end{aligned}
$$


Note that the first of these equations coincides with (36a) (the first discrete equation of the method in Section 4), while the second one is (71b) (the second equation in the method of Section 6.2). The discrete exterior solution is defined with (72). The same kind of manipulations that we have been applied above shows that the pair $\left(u_{h}, u_{h}^{\text {ext }}\right)$ satisfies

$$
\begin{array}{r}
\ddot{u}_{h}=\Delta_{h}^{\kappa} u_{h}-\gamma_{h}^{t} \llbracket \partial_{\nu} u_{h}^{\text {ext }} \rrbracket, \\
\ddot{u}_{h}^{\text {ext }}=\Delta_{ \pm} u_{h}^{\text {ext }}, \\
\llbracket \gamma u_{h}^{\text {ext }} \rrbracket+\gamma u_{h}=0, \\
\gamma^{-} u_{h}^{\text {ext }} \in X_{h}^{\circ}, \\
\llbracket \partial_{\nu} u_{h}^{\text {ext }} \in X_{h},
\end{array}
$$

with the discrete operators defined in (151)-(52). It is simple to see that the transmission conditions above lead to a choice of spaces like (65)-(67), while the operator itself is (53). This mismatch between domain of the operator and operator leads to lack of energy conservation, namely, for smooth solutions

$$
\frac{\mathrm{d}}{\mathrm{d} t}\left(\frac{1}{2}\left\|c^{-1} \dot{u}_{h}\right\|_{\Omega}^{2}+\frac{1}{2}\left\|\kappa^{1 / 2} \nabla u_{h}\right\|_{\Omega}^{2}+\frac{1}{2}\left\|\dot{u}_{h}^{\text {ext }}\right\|_{\mathbb{R}^{d} \backslash \Gamma}^{2}+\frac{1}{2}\left\|\nabla u_{h}^{\text {ext }}\right\|_{\mathbb{R}^{d} \backslash \Gamma}^{2}\right)=\left\langle\partial_{\nu}^{-} u_{h}^{\text {ext }}, \llbracket \gamma \dot{u}_{h}^{\text {ext }} \rrbracket\right\rangle_{\Gamma} .
$$

Furthermore, by recasting the evolution problem as a first order system, it is possible to show that the corresponding operator $\mathcal{A}$ in (89) is not maximal dissipative (cf. [18, Chapter 4]) and therefore it cannot be the infinitesimal generator of a contractive strongly continuous semigroup. As in the case of the discrete Kirchhoff formula of Section 7.1, this does not mean that the discretization leads to an unstable method, but it is at least a hint that some problems might arise due to the lack of energy conservation.

\section{Some abstract arguments about wave equations}

In this section we summarize the abstract results on Cauchy problems for second order equations that we have used throughout the article. The results are elementary consequences of the theory of strongly continuous groups of isometries in the Hilbert setting that can be found in basic texts as [12] or [18].

Cauchy problems for abstract wave equations. Let us consider three Hilbert spaces with continuous inclusions

$$
D(A) \subset V \subset H .
$$

The inner produces and norms of $V$ and $H$ will be recognized with the name of the space as a subscript. Let $A: D(A) \rightarrow H$ be a bounded linear operator satisfying:

(a) an abstract Green's identity

$$
(A u, v)_{H}+(u, v)_{V}=0 \quad \forall u \in D(A) \quad v \in V
$$

(b) a surjectivity condition

$$
I-A: D(A) \rightarrow H \text { is onto, }
$$

$I$ being the inclusion operator. 
Then, for arbitrary $\left(u_{0}, v_{0}\right) \in D(A) \times V$, the initial value problem

$$
\begin{aligned}
\ddot{u} & =A u \quad t \geq 0, \\
u(0) & =u_{0}, \\
\dot{u}(0) & =v_{0},
\end{aligned}
$$

has a unique solution

$$
u \in \mathcal{C}^{2}([0, \infty) ; H) \cap \mathcal{C}^{1}([0, \infty) ; V) \cap \mathcal{C}([0, \infty) ; D(A))
$$

Moreover, the energy

$$
e(t):=\frac{1}{2}\|\dot{u}(t)\|_{H}^{2}+\frac{1}{2}\|u(t)\|_{V}^{2}
$$

is constant as a function of $t \in[0, \infty)$. Also, the injections (84) are dense. The above result can be proved by considering the unbounded operator in $V \times H$

$$
\mathcal{A}:=\left[\begin{array}{ll}
0 & I \\
A & 0
\end{array}\right]: D(\mathcal{A}) \rightarrow V \times H, \quad D(\mathcal{A}):=D(A) \times V \subset V \times H
$$

and showing that $\pm \mathcal{A}$ are maximal dissipative and therefore $\mathcal{A}$ is the infinitesimal generator of a $C_{0}$-group of isometries in the Hilbert space $V \times H$. The possibility of reducing all the hypotheses to properties that have to be satisfied by $A$ is related to the fact that $D(\mathcal{A})$ is the product space $D(A) \times V$, where the second space is the same as the first space in the Hilbert space $V \times H$ where the problem is set.

Problems displaying rigid motions. Assume that we have three spaces in the same conditions above and that in $V$ we also have a seminorm $|\cdot|_{V}$, proceeding from a semiinner product $[\cdot, \cdot]_{V}$. A space of rigid motions of the system (87) is a finite dimensional space $M$ such that

$$
M \subset D(A), \quad M \subset \operatorname{ker}(A), \quad|m|_{V}=0 \quad \forall m \in M,
$$

and, if $P: H \rightarrow M$ denotes the orthogonal projection onto $M$,

$$
C_{1}\|v\|_{V}^{2} \leq|v|_{V}^{2}+\|P v\|_{H}^{2} \leq C_{2}\|v\|_{V}^{2} \quad \forall v \in V .
$$

In particular, this implies that $|v|_{V}=0$ if and only if $v \in M$. The energy of the system is now measured in the following form

$$
e(t):=\frac{1}{2}\|\dot{u}(t)\|_{H}^{2}+\frac{1}{2}|u(t)|_{V}^{2}
$$

If we start with initial conditions $(m, 0) \in M \times\{0\}$, the solution remains constant over time and energy vanishes. If we start with $(0, m) \in\{0\} \times M$, then the solution is $u(t)=m t$. This solution has only kinetic energy and potential energy vanishes identically

$$
e(t)=\frac{1}{2}\|m\|_{H}^{2} .
$$

The conditions are: 
(a') a (modified) abstract Green identity

$$
(A u, v)_{H}+[u, v]_{V}=0 \quad \forall u \in D(A) \quad v \in V
$$

(b) the surjectivity condition $I-A: D(A) \rightarrow H$ is onto.

With these hypotheses, problem (87) has a unique solution with the regularity of (88) for any $\left(u_{0}, v_{0}\right) \in D(A) \times V$. Energy, defined with (91), is constant in time.

Remark 8.1. To see how the frame of evolution equations with rigid motions fits into the general frame, we need to consider the spaces

$$
\begin{gathered}
H_{0}:=\{u \in H: P u=0\}=\left\{u \in H:(u, m)_{H}=0 \quad \forall m \in M\right\}, \\
V_{0}:=V \cap H_{0}, \quad D\left(A_{0}\right):=D(A) \cap H_{0} .
\end{gathered}
$$

In $V_{0}$ we consider the norm $|\cdot|_{V}($ see (90) $)$. The operator $A$ has range in $H_{0}$, since (92) implies that

$$
0=(A u, m)_{H}+[u, m]_{V}=(A u, m)_{H} \quad \forall m \in M .
$$

Finally, the surjectivity condition implies that $I-A_{0}: D\left(A_{0}\right) \rightarrow H$ is surjective by the same argument.

Waves in free space. Problem (44)-(5), that deals with propagation of initial conditions by the wave equation in free space does not fit in the simple frame of this section unless we apply some kind of cut-off argument. The main difficulty stems from the definition of the energy space.

(1) It is well known that the closure of the space of smooth compactly supported functions with the norm $\|\nabla \cdot\|_{\mathbb{R}^{3}}$ is not a subset of $L^{2}\left(\mathbb{R}^{3}\right)$. Instead, the resulting space can be characterized as a weighted Sobolev space (see [16, 13, 2]). The simple frame with three spaces $(D(A)$ as the domain of the operator, $V$ as the potential energy space and $H$ as the space where kinetic energy is measured) cannot be used for the corresponding second order Cauchy problem. Instead, the problem has to be rewritten as a first order system with four Hilbert spaces involved (compare with (89)). Conditions on the operator need to be written in a much more complicated form in order to show existence, uniqueness and energy conservation.

(2) The two dimensional case is even more involved. As explained in [13], the closure of the space of smooth compactly supported functions with $\|\nabla \cdot\|_{\mathbb{R}^{2}}$ cannot be understood as a space of functions in any natural way. This adds another complication to the four space setting that is needed in the three dimensional case.

Since the present work deals with compactly supported initial data and speed of propagation of waves is finite, the strategy of cutting off the analytical domain can be applied for any finite time interval, which is enough for our purposes. 


\section{References}

[1] R. A. Adams and J. J. F. Fournier. Sobolev spaces, volume 140 of Pure and Applied Mathematics (Amsterdam). Elsevier/Academic Press, Amsterdam, second edition, 2003.

[2] C. Amrouche, V. Girault, and J. Giroire. Weighted Sobolev spaces for Laplace's equation in $\mathbf{R}^{n}$. J. Math. Pures Appl. (9), 73(6):579-606, 1994.

[3] A. Bamberger and T. H. Duong. Formulation variationnelle espace-temps pour le calcul par potentiel retardé de la diffraction d'une onde acoustique. I. Math. Methods Appl. Sci., 8(3):405-435, 1986.

[4] A. Bamberger and T. H. Duong. Formulation variationnelle pour le calcul de la diffraction d'une onde acoustique par une surface rigide. Math. Methods Appl. Sci., 8(4):598-608, 1986.

[5] L. Banjai. Multistep and multistage convolution quadrature for the wave equation: algorithms and experiments. SIAM J. Sci. Comput., 32(5):2964-2994, 2010.

[6] L. Banjai, A. R. Laliena, and F.-J. Sayas. A fully discrete kirchhoff formula with cq-bem. In preparation.

[7] L. Banjai, C. Lubich, and J. M. Melenk. Runge-kutta convolution quadrature for operators arising in wave propagation, accepted for publication in numerische mathematik. To appear in Numer. Math.

[8] L. Banjai and S. Sauter. Rapid solution of the wave equation in unbounded domains. SIAM J. Numer. Anal., 47(1):227-249, 2008/09.

[9] M. Costabel. Symmetric methods for the coupling of finite elements and boundary elements. In Boundary elements IX, Vol. 1 (Stuttgart, 1987), pages 411-420. Comput. Mech., Southampton, 1987.

[10] M. Costabel. Boundary integral operators on Lipschitz domains: elementary results. SIAM J. Math. Anal., 19(3):613-626, 1988.

[11] M. Costabel. Time-dependent problems with the boundary integral equation method. In Encyclopedia of Computational Mechanics. Edited by Erwin Stein, Renee de Borst and Thomas Hughes, page Chapter 22. John Wiley, 2003.

[12] K.-J. Engel and R. Nagel. A short course on operator semigroups. Universitext. Springer, New York, 2006.

[13] G. P. Galdi and S. Rionero. Weighted energy methods in fluid dynamics and elasticity, volume 1134 of Lecture Notes in Mathematics. Springer-Verlag, Berlin, 1985.

[14] T. Ha-Duong. On retarded potential boundary integral equations and their discretisation. In Topics in computational wave propagation, volume 31 of Lect. Notes Comput. Sci. Eng., pages 301-336. Springer, Berlin, 2003. 
[15] H. D. Han. A new class of variational formulations for the coupling of finite and boundary element methods. J. Comput. Math., 8(3):223-232, 1990.

[16] B. Hanouzet. Espaces de Sobolev avec poids application au problème de Dirichlet dans un demi espace. Rend. Sem. Mat. Univ. Padova, 46:227-272, 1971.

[17] C. Johnson and J.-C. Nédélec. On the coupling of boundary integral and finite element methods. Math. Comp., 35(152):1063-1079, 1980.

[18] S. Kesavan. Topics in functional analysis and applications. John Wiley \& Sons Inc., New York, 1989.

[19] A. R. Laliena and F.-J. Sayas. A distributional version of Kirchhoff's formula. J. Math. Anal. Appl., 359(1):197-208, 2009.

[20] A. R. Laliena and F.-J. Sayas. Theoretical aspects of the application of convolution quadrature to scattering of acoustic waves. Numer. Math., 112(4):637-678, 2009.

[21] C. Lubich. On the multistep time discretization of linear initial-boundary value problems and their boundary integral equations. Numer. Math., 67(3):365-389, 1994.

[22] W. McLean. Strongly elliptic systems and boundary integral equations. Cambridge University Press, Cambridge, 2000.

[23] F.-J. Sayas. The validity of Johnson-Nédélec's BEM-FEM coupling on polygonal interfaces. SIAM J. Numer. Anal., 47(5):3451-3463, 2009.

[24] M. Schanz. Wave Propagation in Viscoelastic and Poroelastic Continua: A Boundary Element Approach (Lecture Notes in Applied and Computational Mechanics). Springer, 2001. 